\title{
OLD STARBURSTS IN ACTIVE NUCLEI: \\ THE CONNECTION BETWEEN STARBURST \\ AND SEYFERT ACTIVITY.
}

\author{
L. ORIGLIA ${ }^{1}$, A.F.M. MOORWOOD ${ }^{2}$, E. OLIVA ${ }^{3}$ \\ ${ }^{1}$ Torino Observatory, I-10025 Pino Torinese \\ ${ }^{2}$ European Southern Observatory, D-85748 Garching \\ ${ }^{3}$ Arcetri Observatory, I-50125 Firenze
}

Infrared spectra of selected stellar absorption features (CO 2.29, 1.62 and Si $1.59 \mu \mathrm{m}$ ) of a sample of Seyferts and many calibrators (stellar clusters, normal galaxies and well known starbursters) are presented. For HII and Seyferts we also report $\mathrm{Br} \gamma$ measurements.

The measured equivalent widths in old stellar systems and HII galaxies are remarkably similar and do not provide reliable diagnostics for distiguishing young from old/metallic systems. However, the features are much broader (i.e. the dynamical mass is larger) in ellipticals $(200-300 \mathrm{Km} / \mathrm{s})$ than in spirals and $\mathrm{HII}$ galaxies $(100-150 \mathrm{Km} / \mathrm{s})$ of similar luminosity.

A more sensitive age indicator is therefore the light to mass ratio $\left(L_{H} / M\right)$ one infers from the observed $\mathrm{H}(1.65 \mu \mathrm{m})$ stellar luminosities and line velocity dispersions. Compared to optical lines (e.g. the CaII triplet) our method has the advantage of providing a direct measurement of the non-stellar contribution to the observed luminosity (which is important in Sy1's), and of minimizing the reddeding correction.

All HII galaxies and several Sy2's exhibit $\mathrm{L}_{\mathrm{H}} / \mathrm{M}>3$ and $\sim 5$ times those of ellipticals and spirals, $\mathrm{Br} \gamma$ spectra indicate that the starbursts in Sy2's are older than in HII galaxies. The $\mathrm{L}_{\mathrm{H}} / \mathrm{M}$ of Sy1's is similar to that of spirals and typical of old disk/bulge populations.

These results are therofore compatible with an $\mathrm{HII} \rightarrow \mathrm{Sy} 2 \rightarrow \mathrm{Sy} 1$ evolutionary scheme where the two Seyfert types are intrinsically different objects. 